\title{
Electrochemical synthesis and characterization of hierarchically branched ZnO nanostructures on ensembles of gold nanowires
}

\author{
Michael Ongaro ${ }^{\mathrm{a}}$, Arianna Gambirasi ${ }^{\mathrm{a}, \mathrm{b}}$, Monica Favaro $^{\mathrm{b}}$, Paolo Ugo ${ }^{\mathrm{a}, *, 1}$ \\ a Department of Molecular Sciences and Nanosystems, University Ca' Foscari of Venice, S. Marta 2137, Venice, Italy \\ ${ }^{\mathrm{b}}$ Institute of Surface and Inorganic Chemistry, Consiglio Nazionale delle Ricerche, Corso Stati Uniti, 435127 Padova, Italy
}

\section{A R T I C L E I N F O}

\section{Article history:}

Received 3 April 2012

Received in revised form 14 June 2012

Accepted 16 June 2012

Available online 1 July 2012

\section{Keywords:}

Zinc oxide

Hierarchical nanostructure

Nanoelectrode

Photooxidation

Electron back scatter diffraction

\begin{abstract}
A B S T R A C T
This study presents an electrosynthetic methodology to obtain hierarchically structured ZnO electrodes with improved surface area, by exploiting gold nanowires ensembles (3D-NEEs) as the growing substrate. By this way, semiconductor electrodes organized in the shape of fir-like branches are obtained. Branched nanofibres are characterized by electron microscopy and electron backscatter diffraction (EBSD), the latter technique allowing the determination of the crystalline habit of individual nanostructures. The hierarchical branched nanowires show enhanced performances with respect to water photooxidation in comparison with already known nanostructured materials such as $1 \mathrm{D}-\mathrm{ZnO}$ nanowires.
\end{abstract}

(c) 2012 Elsevier Ltd. All rights reserved.

\section{Introduction}

$\mathrm{ZnO}$ is a semiconductor characterized by a wide band gap $(3.37 \mathrm{eV})$ with large exciton binding energy (60 meV) [1]; it finds application in many fields such as photovoltaics, gas sensors, photocatalysis, energetics, optics, and photoelectrochemical cells. Thanks to its superior electron mobility, the ease of crystallization and possibility of anisotropic growth [2], ZnO is considered a suitable substitute for $\mathrm{TiO}_{2}$. Moreover, $\mathrm{ZnO}$ can be prepared in a variety of morphologies such as: nanorods [3], nanobelts, nanosaws [4], nanosprings, nanorings [5], nanostars [6]. The nanostructuration of this semiconductor, in particular, quasi 1-D nanostructures (nanowires, NWs) are considered promising for photoelectrochemical applications because these structures are expected to further increase the electron diffusion length in photoelectrode films, so providing a direct charge transport pathway for the photogenerated electrons [7-9]. However, a limit in the photoelectrochemical performances of linear nanostructures can come from the relatively low surface area of 1D structures, in comparison with other morphologies. Recently, many efforts were put on the integration of 1D nanoscale building blocks into 3D architectures. The goal is to develop hierarchical structures in which the surface area of the semiconductor is enhanced, while keeping the electron transport

\footnotetext{
* Corresponding author. Tel.: +39 041 2348503; fax: +39 0412348594.

E-mail address: ugo@unive.it (P. Ugo).

1 ISE member.
}

efficiency as high as possible. Shen et al. [10] reported the growth of a number of hierarchical structures, such as $\mathrm{ZnO}$ nanocones or nanobottles. Some of these complex structures are obtained by exploiting physical methods of preparation, such as chemical vapor deposition (CVD), plasma enhanced chemical vapor deposition (PECVD) and thermal evaporation; however, such methods require expensive instrumentation, are energy consumptive and are not suitable for large-scale production. Hydrothermal growth is considered a good candidate for low cost production of nanostructured $\mathrm{ZnO}$ materials, but this technique can be time consuming since a seeding layer deposition step is required [3]. Cathodic electrodeposition has been recognized as one of the most effective methods to prepare nanomaterials since it is simple and economic, does not require vacuum or extreme temperature and can be easily scaled up. By changing the process parameters (i.e. applied potential, concentration and $\mathrm{pH}$ of the electrolyte, operating temperature and deposition time), the film morphology and thickness can be controlled [11]. Electrochemical deposition methods are based on the fast and cheap cathodic deposition of ZnO onto a suitable working electrode substrate. Usually, the mechanism is based on the reduction of a precursor such as nitrate [12] (see Eq. (1)), hydrogen peroxide [13] (see Eq. (2)) or dissolved $\mathrm{O}_{2}$ [14] (see Eq. (3)), all these three reactions produce at the electrode/solution interface a large $\mathrm{OH}^{-}$concentration:

$$
\begin{aligned}
& \mathrm{NO}_{3}{ }^{-}+\mathrm{H}_{2} \mathrm{O}+2 \mathrm{e}^{-} \rightarrow \mathrm{NO}_{2}^{-}+2 \mathrm{OH}^{-} \\
& \mathrm{H}_{2} \mathrm{O}_{2}+2 \mathrm{e}^{-} \rightarrow 2 \mathrm{OH}^{-}
\end{aligned}
$$


$\mathrm{O}_{2}+2 \mathrm{H}_{2} \mathrm{O}+4 \mathrm{e}^{-} \rightarrow 4 \mathrm{OH}^{-}$

The local generation of hydroxyl anions causes, on the electrode surface, the precipitation of $\mathrm{Zn}(\mathrm{OH})_{2}$ which, at relatively high temperatures $\left(>34^{\circ} \mathrm{C}\right)$ [15], is dehydrated to $\mathrm{ZnO}$ (Eq. (4)). Since the growth of the semiconductor on the [ $\left.\begin{array}{lllll}0 & 0 & 0 & 1\end{array}\right]$ plane of the $\mathrm{ZnO}$ crystals is faster, a deposit with the shape of nanorods or nanowires is typically obtained [16].

$\mathrm{Zn}^{2+}+2 \mathrm{OH}^{-} \rightarrow \mathrm{ZnO}+\mathrm{H}_{2} \mathrm{O}$

If a nanostructured electrode is employed as the substrate, a hierarchical semiconductor electrode can be obtained. For instance Xu et al. $[17,18]$ for example developed a synthetic route for the preparation of twofold and sixfold hierarchical structures, by multiple electrochemical depositions on transparent electrodes. More recently, Elias et al. [19] developed a synthetic approach, based on $\mathrm{O}_{2}$ reduction, to fabricate ordered hollow urchin-like $\mathrm{ZnO}$ NWs grown on a multilayer of polystyrene micro-spheres used as the templating substrate.

In the present work we focus on the electrochemical growth of $\mathrm{ZnO}$ nanowires (NWs), onto ensembles of gold nanowires ( $\mathrm{Au}$ NWs); the final product of the process being an ensemble of hierarchically branched $\mathrm{ZnO}-\mathrm{Au}$ nanostructures. The preparation sequence is summarized in Scheme 1.

At first, an ensemble of Au nanoelectrodes is obtained by membrane template deposition $[20,21]$, then, it is etched to expose the nanowires $[22,23]$ which are finally used as the substrate for the electrochemical growth of the $\mathrm{ZnO}$ nanofibres. By this way, hierarchically built nanostructures are generated, in which the ZnO NWs were grown as fir needles on Au nanobranches. As said above, the goal is to obtain nanomaterials with improved photoelectrochemical performances.

At the best of our knowledge this is the first example of combined use of membrane template deposition of 1-D nanostructures, with electrochemically directed branching of semiconductor subnanostructures.

One problem encountered with the characterization of so small and complex nanomaterials is the determination of the crystal structure of the individual nanostructure. To this aim we apply the electron back scatter diffraction (EBSD) technique, which allows one to perform the crystallographic analysis with high spatial resolution [24]. Very recently, one of the present authors proved that EBSD can be applied to the characterization of individual copper nanowires [25]; now, we demonstrate the possibility to extend

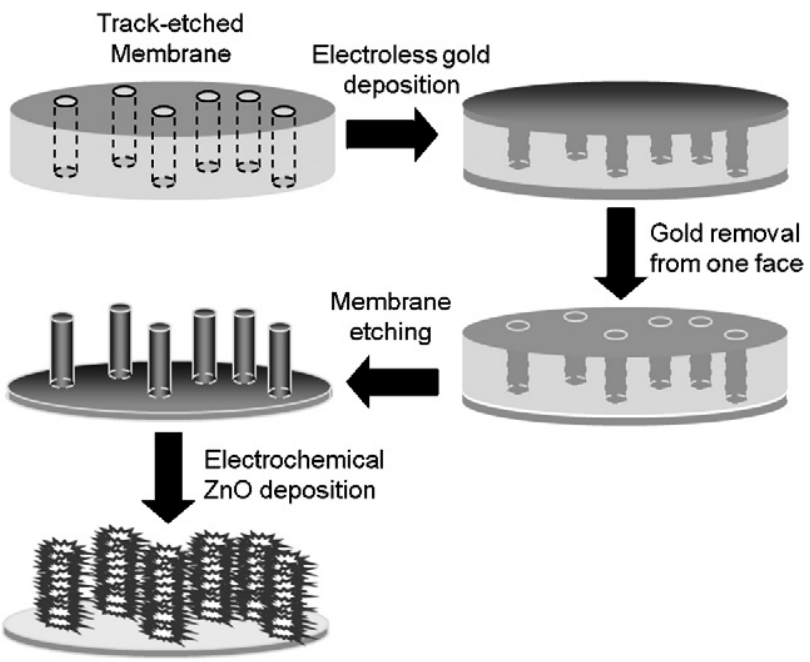

Scheme 1. Preparation of $\mathrm{ZnO}$ branched nanowires. the use of this powerful characterization technique to the hybrid hierarchical nanostructures here prepared.

\section{Experimental}

\subsection{Electrochemical and photoelectrochemical apparatus}

All the electrochemical analysis and depositions were performed using a $\mathrm{CH} 660 \mathrm{~B}$ potentiostat. The photoelectrochemical water oxidation experiments were conducted in a three electrode quartz cell. UV light irradiation was performed using three ' $T L$ ' 20W/05 PHILIPS UV-lamps.

\subsection{FEG-ESEM (field emission gun environmental scanning electron microscopy) and electron backscatter diffraction analysis}

SEM and FEG-ESEM analyses were performed with a tabletop Hitachi TM3000 SEM and with a FEI Quanta FEG-ESEM, respectively. EBSD analyses were conducted in the FEI Quanta FEG-ESEM microscope operating at an accelerating voltage of $20 \mathrm{kV}$, with a working distance of $10 \mathrm{~mm}$ and the sample tilted of $75^{\circ}$ to horizontal. The EBSD facility included the EDAX-TSL DigiView detector consisting of a DVC-1412M digital camera with CCD sensor and a circular phosphor screen as intensifier. The TSL software OIM $5.31^{\mathrm{TM}}$ package was used to collect, index and analyze EBSD patterns.

\subsection{Template fabrication of NEEs}

Specific details, related to the synthetic procedure and to the electrochemical and optical properties of NEEs, can be found in the first original papers $[20,23,26,27]$ as well as in more recent reviews $[21,28,29,27]$. Briefly, polycarbonate filtration membranes (SPIPore, $47 \mathrm{~mm}$ filter diameter, $6 \mu \mathrm{m}$ filter thickness, $6 \times 10^{8}$ pores $\mathrm{cm}^{-2}$ ) with a nominal pore diameter of $80 \mathrm{~nm}$ were used as template to prepare 3D-NEEs. After sensitizing the polycarbonate membrane by immersion into a $0.026 \mathrm{M} \mathrm{SnCl}_{2}$ solution and $0.07 \mathrm{M} \mathrm{CF}_{3} \mathrm{COOH}$ in 50:50, methanol/water for $45 \mathrm{~min}$ the membrane was immersed in a $0.029 \mathrm{M} \mathrm{Ag}\left[\left(\mathrm{NH}_{3}\right)_{2}\right] \mathrm{NO}_{3}$ solution for $10 \mathrm{~min}$. Afterward the membrane was immersed into a gold plating bath $\left(7.9 \times 10^{-3} \mathrm{M} \mathrm{Na}_{3}\left[\mathrm{Au}\left(\mathrm{SO}_{3}\right)_{2}\right]\right.$ and $\left.0.127 \mathrm{M} \mathrm{Na}_{2} \mathrm{SO}_{3}\right)$ which was kept at $0^{\circ} \mathrm{C}$. After $20 \mathrm{~min} 1 \mathrm{~mL}$ of formaldehyde (37\%) was added and after $1 \mathrm{~h}$ an additional $\mathrm{mL}$ of formaldehyde (37\%) was added. The deposition was then allowed to proceed for another $23 \mathrm{~h}$ after which the membrane was rinsed in deionized water and immersed in $\mathrm{HNO}_{3} 10 \%$ for $12 \mathrm{~h}$. The membrane was finally rinsed in water and dried, resulting in the deposition of continuous gold nanowires within the pores and on both sides of the membrane faces. The smooth side of the membrane was peeled off with adhesive tape $3 \mathrm{M}$ (scotch Magic ${ }^{\mathrm{TM}}$ ) so that the tips of the nanowires remain exposed on one side. All the nanowires are interconnected to each other by a gold back layer which acts as current collector. Afterwards the membrane was etched with oxygen plasma to completely remove the polycarbonate, obtaining the so called 3DNEEs. The following etching conditions have been used: oxygen flux $100 \mathrm{~mL} / \mathrm{min}$, power $700 \mathrm{~W}$, etching time $60 \mathrm{~min}$. Fig. 1 shows the SEM image of an ensemble of $80 \mathrm{~nm}$ diameter of Au NWs.

The gold "nanobrush" was assembled to obtain an electrode as sketched in Fig. 2 according to the following procedure. A piece of copper adhesive tape $(5 \times 60 \mathrm{~mm})$ with conductive glue (Ted Pella, Inc.) is first affixed on a small adhesive non conductive aluminum square and then to a $5 \mathrm{~mm} \times 5 \mathrm{~mm}$ piece of gold "brush", so that only a small part is in contact with the copper tape, this is because the conductive glue on the copper tape contains Ni particles which could damage the gold layer [20]. Finally strips of non conductive tape are applied to the lower and upper side of the assembly in order 


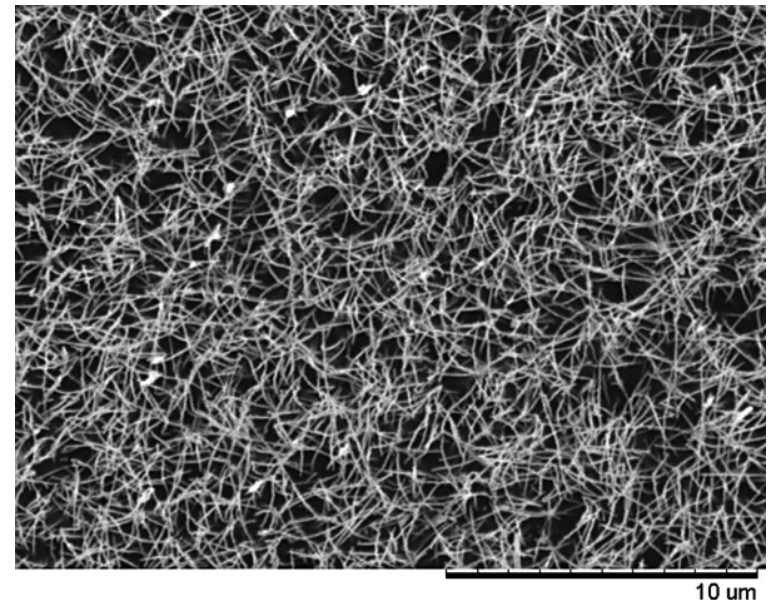

Fig. 1. SEM image of 3D-NEE obtained by electroless gold deposition on tracketched polycarbonate membrane with nominal pore diameter of $80 \mathrm{~nm}$, after oxygen plasma treatment.

to insulate the aluminum and copper tape. A circular hole with an area of $0.07 \mathrm{~cm}^{2}$ is punched into the upper piece of insulator prior to its placement on the assembly. This hole defines the geometric area of the ensembles, that is the area exposed to the solution.

\subsection{Optimization of $\mathrm{ZnO}$ deposition parameters}

The electrochemical system involved in the $\mathrm{ZnO}$ deposition has been studied by cyclic voltammetry (CV). The analysis was performed in a thermostated three electrodes cell, at $85^{\circ} \mathrm{C}$ in a $5 \mathrm{mM} \mathrm{Zn}\left(\mathrm{NO}_{3}\right)_{2}$ and $5 \mathrm{mM}$ hexamethylenetetramine $\left(\mathrm{C}_{6} \mathrm{H}_{12} \mathrm{~N}_{4}\right.$,

(A)

(B)

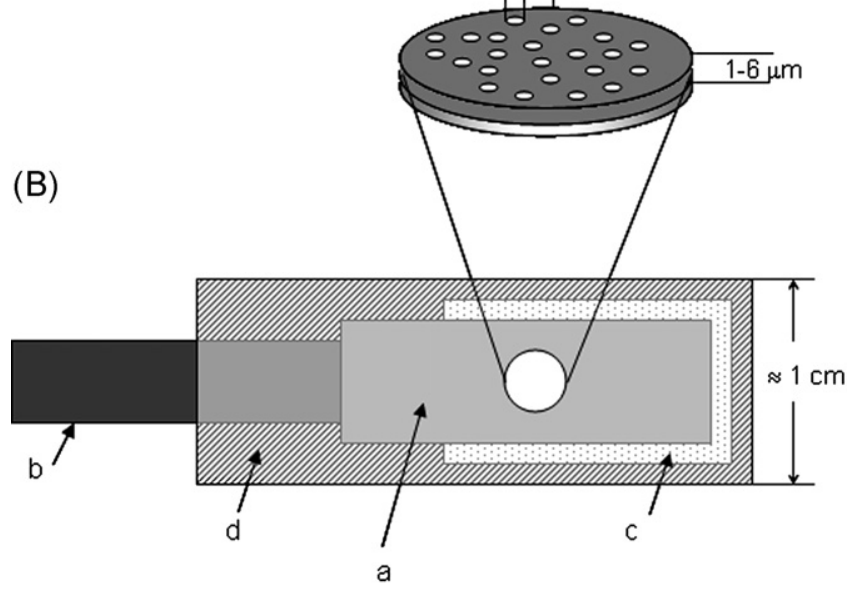

(C)

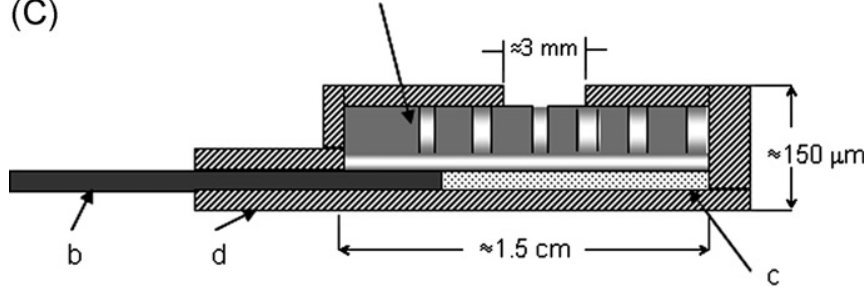

Fig. 2. Scheme of an Au-NEE prepared using a track-etched polycarbonate membrane as template. (A) Particular of the section of the active area; (B) top view; (C) section of the all NEE ready for use as working electrode. (a) Track-etched golden membrane; (b) Copper adhesive tape with conductive glue to connect to instrumentation; (c) Aluminum adhesive foil with non-conductive glue; (d) Insulating tape. Not all details (namely, the nanoelectrodes dimension) are in scale.
HMTA) aqueous solution. The reagents were used as received from Alfa Aesar. Both ITO (Delta technologies, Indium Tin Oxide coated glass, Rs $=4-8 \Omega$ ) electrodes and 3D-NEEs were used as working electrode, an $\mathrm{Ag} / \mathrm{AgCl} \mathrm{KCl}$ saturated electrode was the reference electrode and a platinum coil was the counter electrode. In this article, all potentials are quoted vs. $\mathrm{Ag} / \mathrm{AgCl} \mathrm{KCl}$ saturated electrode. The potential was scanned from $0 \mathrm{~V}$ to $-0.800 \mathrm{~V}$ with a scan rate $10 \mathrm{mV} / \mathrm{s}$. In order to evaluate the effect of dissolved oxygen, the voltammetric analysis was performed before and after deoxygenating the solution. The dissolved oxygen was removed by bubbling nitrogen in the solution for $30 \mathrm{~min}$ and performing the experiments under a nitrogen atmosphere.

\subsection{Semiconductor electrode fabrication}

$\mathrm{ZnO}$ electrodeposition experiments were performed using the above reported conditions, operating at a constant potentials of $-0.550 \mathrm{~V}$ or $-0.700 \mathrm{~V}$. Gold nanowires arrays and ITO electrodes were alternatively used as the working electrode. Also the electrodeposition experiments were conducted in the presence and in the absence of dissolved oxygen. At the end of the growth time (typically $30 \mathrm{~min}$ ) the working electrode was removed from the solution and immediately rinsed with deionized water to remove any residual salt from the surface.

Eight different samples, one for each deposition condition and substrate, were obtained and labeled using the format "XXXYYYZZ", where "XXX" defines the substrate, i.e. ITO or NEE, YYY defines the deposition potential that is 550 if the deposition was conducted at $-0.550 \mathrm{~V}$ and 700 if it was conducted at $-0.700 \mathrm{~V}$ and finally " $\mathrm{ZZ}$ " defines if the deposition was performed in presence of oxygen" $\mathrm{O}_{2}$ " or in deoxygenated solution " $\mathrm{N}_{2}$ ". For example, $\mathrm{ZnO}$ NWs grown on $3 \mathrm{D}-\mathrm{NEE}$ at a potential of $-0.700 \mathrm{~V}$ in deoxygenated solution would be labeled NEE700N2.

\subsection{Photoelectrochemical experiments}

Amperometric $I-t$ curves were recorded applying a constant potential of $0.400 \mathrm{~V}$ in $0.1 \mathrm{M}$ phosphate buffer solution $\mathrm{pH}$ 7.0. The current was measured for the first thirty seconds in the dark, then the lamps were turned on and the electrode was irradiated with UV light with an irradiance of $6 \mathrm{~W} / \mathrm{m}^{2}$.

\section{Results and discussion}

\subsection{Electrochemical $\mathrm{ZnO}$ deposition}

Preliminary CV experiments showed that at the electrodes studied here, oxygen reduction takes place at $-0.450 \mathrm{~V}$ while nitrate reduction occurs at approximately $-0.700 \mathrm{~V}$ on ITO and at $-0.500 \mathrm{~V}$ on 3D-NEE (not shown). The observed anticipation of the $\mathrm{NO}_{3}{ }^{-}$reduction potential at the 3D-NEE suggests the operativity of a catalytic effect on this process, related to the fact that the Au-nanowires, which compose the NEE, present indeed a subnanomorphology made of merging gold nanoparticles. It is indeed known that gold nanoparticles are good electrocatalyst for nitrate reduction.

On the basis of these preliminary results, a series of experiments was performed in order to study the effect of the applied potential on the morphology of the deposit formed. To this aim, two different potentials, namely $-0.550 \mathrm{~V}$ and $-0.700 \mathrm{~V}$, were applied to

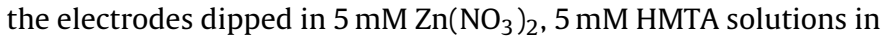
the presence and absence of dissolved oxygen. Following previous reports [12], HMTA was added as nucleation control agent; it was demonstrated that HMTA releases hydroxyl ions and acts as organic soft template at high temperature in aqueous solution [16]. 


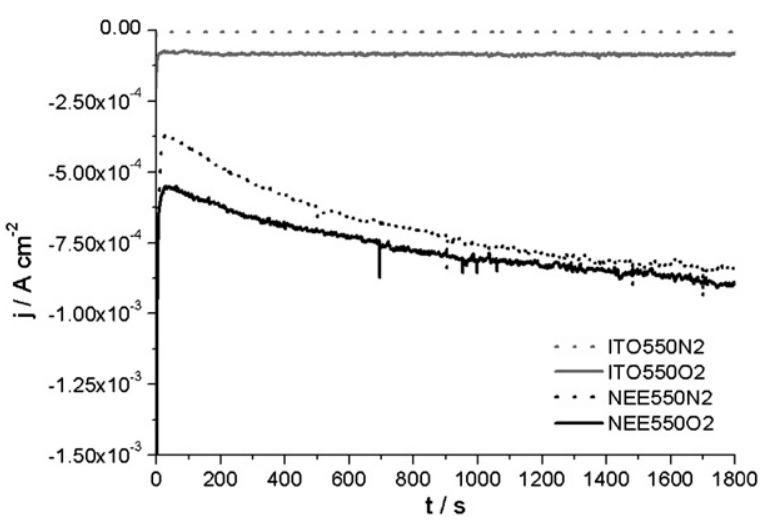

Fig. 3. Chronoamperograms of $\mathrm{ZnO}$ depositions on ITO electrode and on 3D-NEE

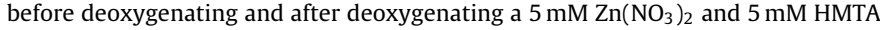
electrolyte solution. Constant potential bias $-0.550 \mathrm{~V}$ at $85^{\circ} \mathrm{C}$.

Fig. 3 reports the comparison between the chronoamperograms recorded when a constant potential of $-0.550 \mathrm{~V}$ was applied at a 3DNEE or at an ITO. The electrodes used in these experiments were labeled as described in Section 2.5: ITO550N2 (gray dotted line), ITO550O2 (gray straight line), NEE550N2 (black dotted line) and NEE550O2 (black straight line). The curve recorded at the ITO550N2 does not show any cathodic current, while at IT055002 a constant current of about $10 \mu \mathrm{Acm}^{-2}$ was detected. This evidence confirms that, at $-0.550 \mathrm{~V}$ on ITO, oxygen reduction occurs.

On the other hand, on the 3D-NEE, a cathodic current is present also in deoxygenated solution, confirming that the nitrate reduction is anticipated on this substrate. One can note that the current at the beginning of the deposition is higher for the NEE550O2; this evidence suggests that, at the NEE at $-0.550 \mathrm{~V}$, both the reduction of nitrate and dissolved $\mathrm{O}_{2}$ occur. It is worth pointing out that the progressive current increase observed in these two latter experiments agrees with a progressive increase of the surface area with deposition time. Finally, the achievement of a final plateau current suggests the operativity of a mass transport diffusive limitation, in agreement with previous studies performed on 3D-NEEs [23]. It can be seen that the deposition current at the 3D-NEE is significantly higher than the one recorded at the flat electrode because of the higher surface area of the former.

Fig. 4 shows the chronoamperograms recorded when a constant potential of $-0.700 \mathrm{~V}$ is applied. As far as the deposition on the ITO electrode is concerned, a cathodic current is detected both on ITO700N2 (gray dotted line) and IT070002 (gray straight line), so confirming that at such a potential, both nitrate and

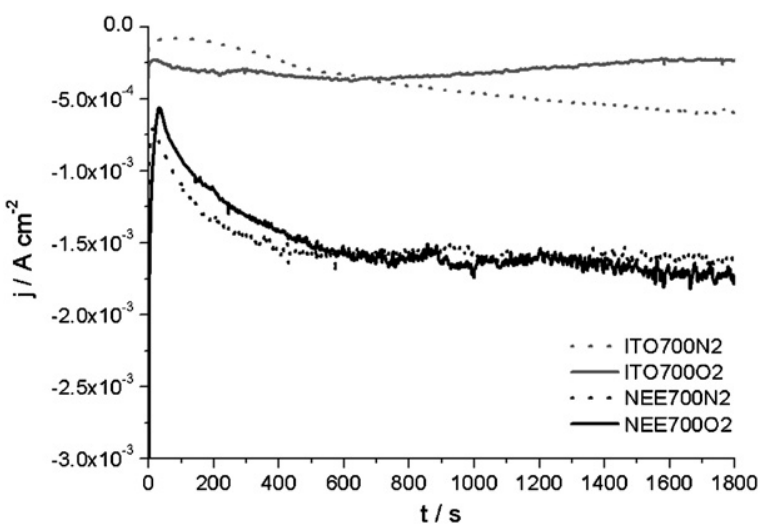

Fig. 4. Chronoamperograms of $\mathrm{ZnO}$ depositions on ITO electrode and on 3D-NEE

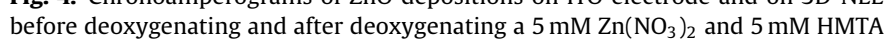
electrolyte solution. Constant potential bias $-0.700 \mathrm{~V}$ at $85^{\circ} \mathrm{C}$. oxygen reduction are operative. In agreement with the possible concomitant occurrence of both reduction processes, the current at the beginning of the deposition is higher for ITO70002. A difference correlated with the presence/absence of dissolved oxygen is that the cathodic current on ITO700O2 is quite constant during the whole deposition time, while on ITO700N2 the current keeps increasing, suggesting a significant increase of the active area during the reduction.

The chronoamperograms, recorded on 3D-NEE in the presence and absence of oxygen, almost overlap, indicating that, at this potential, the presence of oxygen does not affect significantly the deposition process; the deposition process now appears to be triggered by the nitrate reduction reaction. For both aerated and de-aerated solutions, at the 3D-NEE, the cathodic current increases with time and achieves a plateau after $600 \mathrm{~s}$, suggesting again a diffusion controlled limitation related to the operativity, at the 3DNEE, of a total overlap diffusion regime [23].

\subsection{Morphological and structural characterization}

The morphological characterization of the different nanostructures obtained in the experimental conditions of Figs. 3 and 4, was performed by SEM and FEG-ESEM analyses. Relevant images, reported in Figs. 5 and 6 , indicate that at both ITO and 3DNEE, nanowires and nanorods are formed, however with different geometries and morphologies which depend on the experimental conditions adopted (i.e. applied potential and presence/absence of dissolved oxygen).

A common feature for the nanostructures in Figs. 5b-d and 6a-d is that EDS analysis data (not-shown), detected zinc and oxygen as the main constituent elements, so allowing us to identify the deposit as $\mathrm{ZnO}$. As far as ITO550N2 is concerned, as expected on the basis of the relevant chronoamperogram in Fig. 3, no deposit is visible. For all the other deposition conditions, NWs with randomly distributed orientation were grown, both on ITO 3D-NEE. On ITO55002 and ITO700N2 the diameter of the deposited NWs is nearly the same, that is, approximately, $200-300 \mathrm{~nm}$, while on IT070002 the average diameter of the NWs is $600 \mathrm{~nm}$.

These results are apparently in contrast with the model presented very recently by Khajavi et al. [30], which states that an higher deposition current (and, consequently, an higher $\mathrm{pH}$ at the electrode surface), leads to thinner ZnO nanorods. Actually, it must be stressed that in our synthetic route the electrodes were not precovered by a $\mathrm{ZnO}$ seed layer deposited by spray pyrolysis at $350^{\circ} \mathrm{C}$, as done in Ref. [30]. Elias et al. [31] demonstrated indeed that the presence of such a layer (named "buffer layer") plays an important role in the nucleation process in the first steps of the deposition. These evidences indicate that in the absence of nanocrystals previously deposited on the substrate (buffer layer), the cathodic current density in the first minutes of the deposition is crucial in defining the morphology of the NWs, since a higher current means larger ZnO crystallites and, subsequently, thicker fibers.

ZnO NWs on NEE550N2 and NEE550O2 grow directly from the gold nanowires which constitute the backbone for a hierarchically branched architecture. The $\mathrm{ZnO}$ needles are $60-70 \mathrm{~nm}$ in diameter and almost $1 \mu \mathrm{m}$ in length (Fig. 8a), hence with significantly smaller diameter than NWs grown on flat ITO electrodes $(200-300 \mathrm{~nm})$, operating under the same deposition conditions. Such a difference in morphology can be explained by a constrain effect, in which the lateral growth of the metal oxide is limited by the gold nanowires diameter and density. Beyond geometrical effects, one of the reason leading to higher aspect ratio $\mathrm{ZnO}$ rods on 3D-NEE substrate, can be related also to the fact that the gold nanowires are made of merged gold nanoparticles [27]. It was indeed demonstrated that gold nanoparticles play a catalytic effect on $\mathrm{ZnO}$ growth and on the morphology and orientation of $\mathrm{ZnO}$ nanofibres [32], leading to 

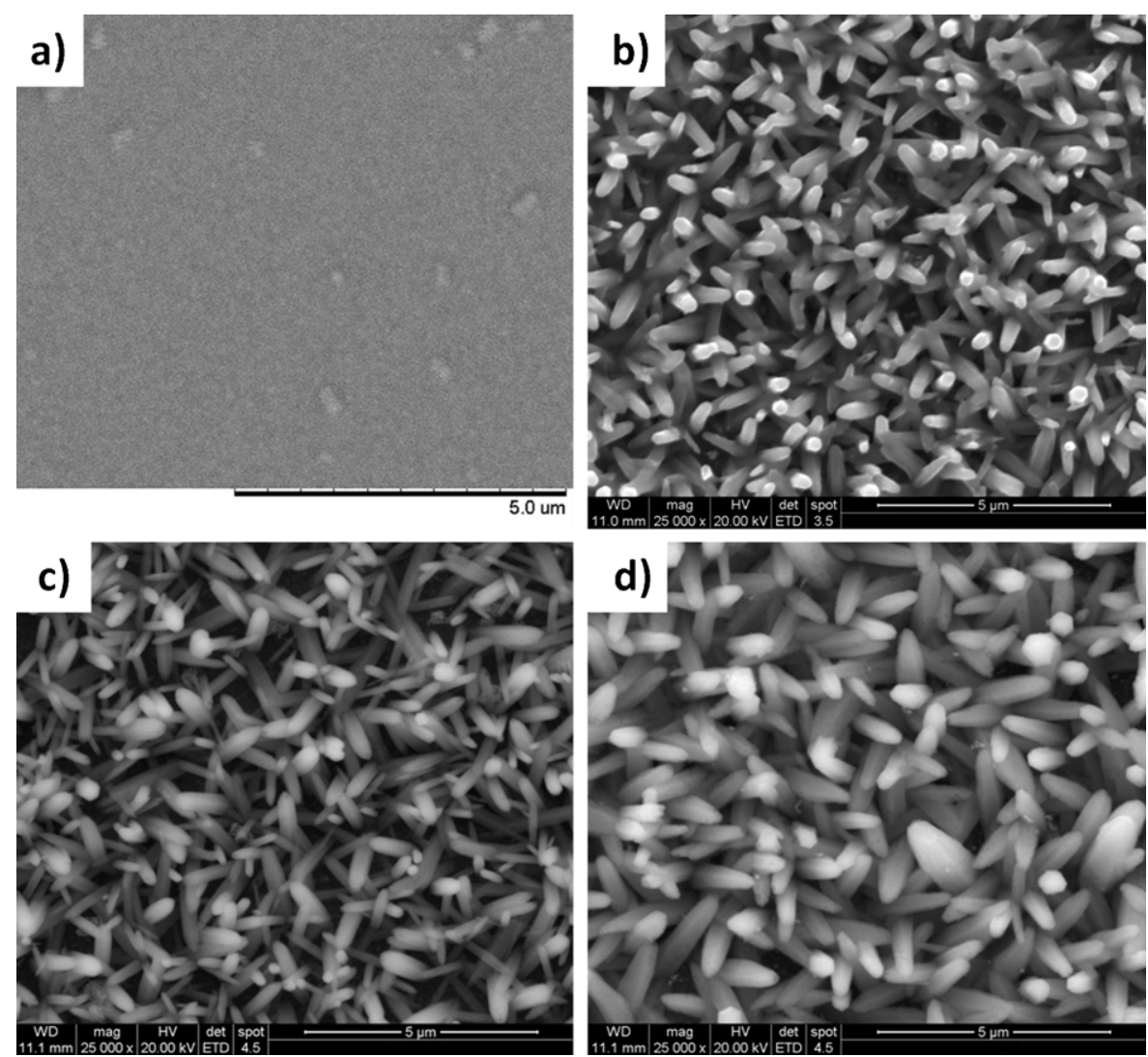

Fig. 5. FEG-ESEM images of ITO electrodes after $30 \mathrm{~min} \mathrm{ZnO}$ electrodeposition using: a deposition potential of $-0.550 \mathrm{~V}$ in the absence (a, ITO550N2) and in the presence of dissolved oxygen (b, IT055002); a deposition potential of $-0.700 \mathrm{~V}$ in the absence (c, ITO700N2) and in the presence of dissolved oxygen (d, ITO70002).
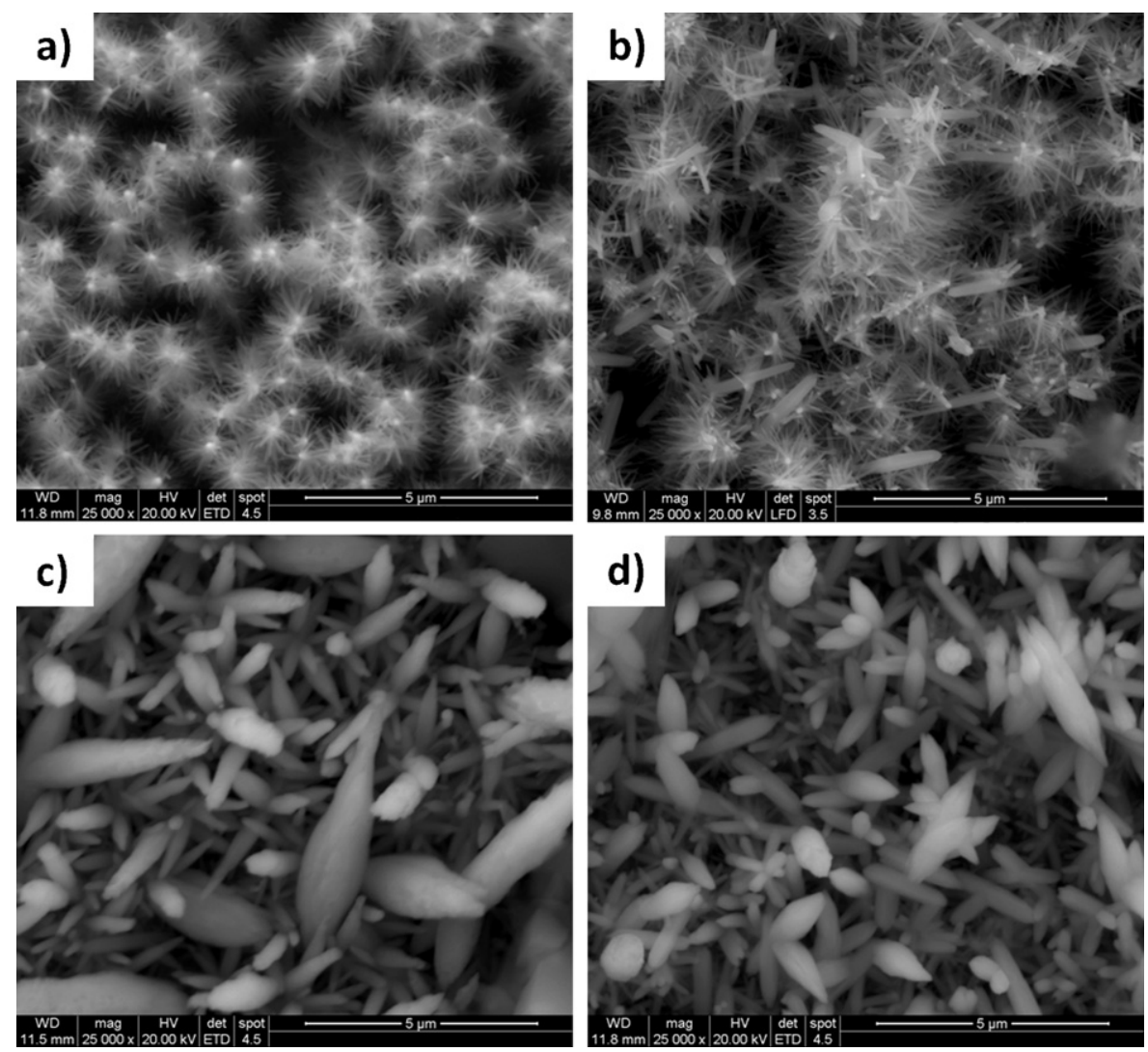

Fig. 6. FEG-ESEM images of 3D-NEE electrodes after $30 \mathrm{~min}$ ZnO electrodeposition using: a deposition potential of $-0.550 \mathrm{~V}$ in the absence (a, NEE550N2) and in the presence of dissolved oxygen (b, NEE55002); a deposition potential of $-0.700 \mathrm{~V}$ in the absence (c, NEE700N2) and in the presence of dissolved oxygen (d, NEE700O2). 

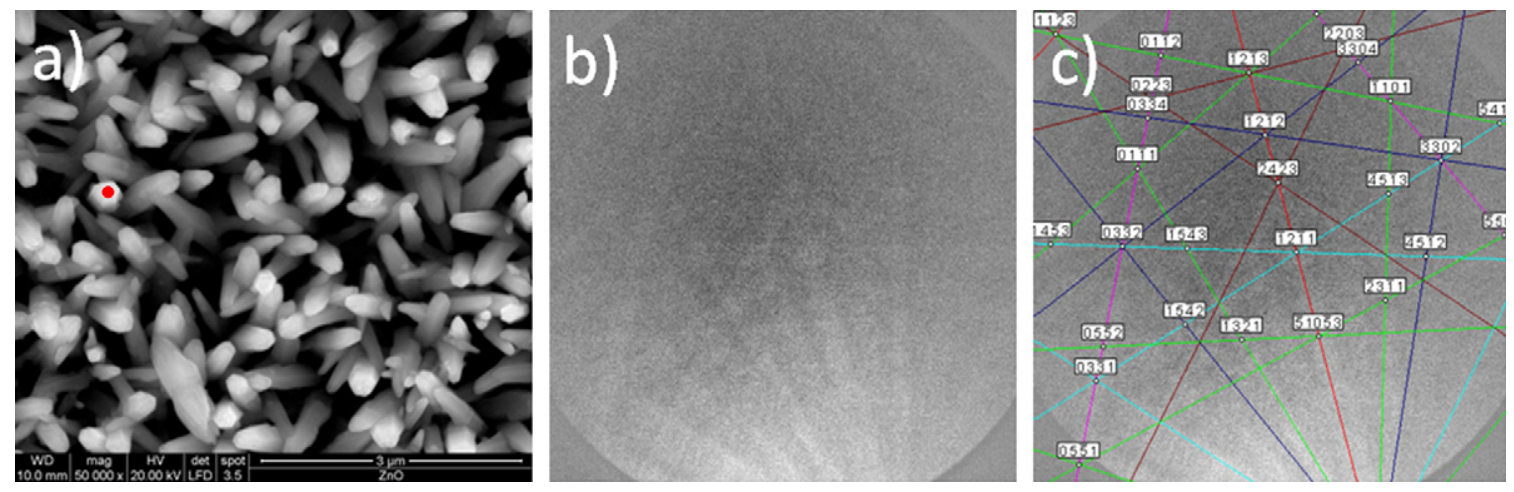

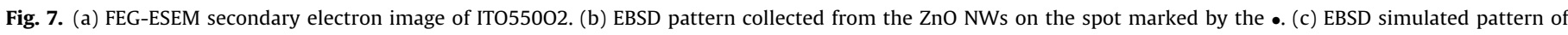
hexagonal wurtzite structure overlaid on pattern of (b).

thinner, longer and more parallel aligned nanorods than on other substrates. Note that, with respect to the $\mathrm{ZnO}$ nanobranches on $\mathrm{ZnO}$ NWs described by Baxter and Aydil [33], our ZnO subnanostructures are approximately 10 times longer, with only 3 times higher diameter. It's also possible to note that the NWs on NEE550O2 are longer and thicker than the ones on NEE550N2, in accordance with a higher charge measured from the chronoamperometric curve.

The SEM pictures referring to NEE700O2 and NEE700N2 show overgrown NWs without a defined morphology. Apparently a too fast deposition leads to a disordered growth, because a too high concentration of $\mathrm{OH}^{-}$is generated on the surface of the electrode, so disrupting the hierarchical growth mechanism.

The crystalline phase identification was performed by EBSD on the most interesting samples in terms of morphology and photoactivity, namely ITO550O2 and NEE550O2. This characterization technique, suitable for any crystalline material, was developed in the 1980s and allows one to obtain crystallographic information such as individual grain orientations, local texture, point-to-point orientation correlations and phase identification and distributions [34].

Even if it was proved that EBSD allows phase identification of submicrometer crystalline particles $[35,36]$ and permits to determine the crystallographic orientation of nanostructures $[37,38]$ there are still few applications of this technique to the characterization of nanostructures [39].

In this study the EBSD patterns were acquired in situ on $\mathrm{ZnO}$ NWs grown on flat ITO electrode, and on ZnO NWs grown from the gold nanowires. The fibers were sampled on different spots of the surface at their top head in order to avoid pattern shadowing by neighboring fibers; note that such an effect could occur in the present samples because their morphology made of a very dense ensemble of fibers. The collected EBSD patterns were compared with the reference patterns calculated for the possible crystallographic phase that, for the elemental composition of the fibers investigated, are hexagonal wurtzite structure (i.e. zincite), and cubic zinc blende structure.

The EBSD patterns collected both from ITO55002 and NEE550O2, result correctly indexed with the pattern calculated for the hexagonal wurtzite structure of zincite (Figs. 7b, c and 8b, c).

\subsection{Photoelectrochemical water oxidation activity}

In order to evaluate the effect of the nanostructuration of $\mathrm{ZnO}$ NWs on the photocatalytic properties of the material, we performed the compared photoelectrochemical characterization of $\mathrm{ZnO} N W s$ grown both on ITO and on 3D-NEE. To this aim we studied the photoelectrochemical oxidation of water. For the sake of clarity, only photocurrents generated at ITO55002 (dotted line) and NEE550O2 (straight line) are reported in Fig. 9.

The current measured under UV irradiation includes the current related to the photoelectrochemical oxidation at the semiconductor electrode plus the double layer charging current and net photocatalyzed residual currents. Since also charging and residual currents scale with the surface area of the working electrode, it is necessary to evaluate the contribution of these currents, in order to extract the real photoelectrochemical contribution from the overall current. For this reason, a preemptive polarization measurement in the dark was performed (first $30 \mathrm{~s}$ on the plots), afterwards, the UV lights were turned on and the photocurrent was recorded. The final photocurrent values taken into account are indeed the
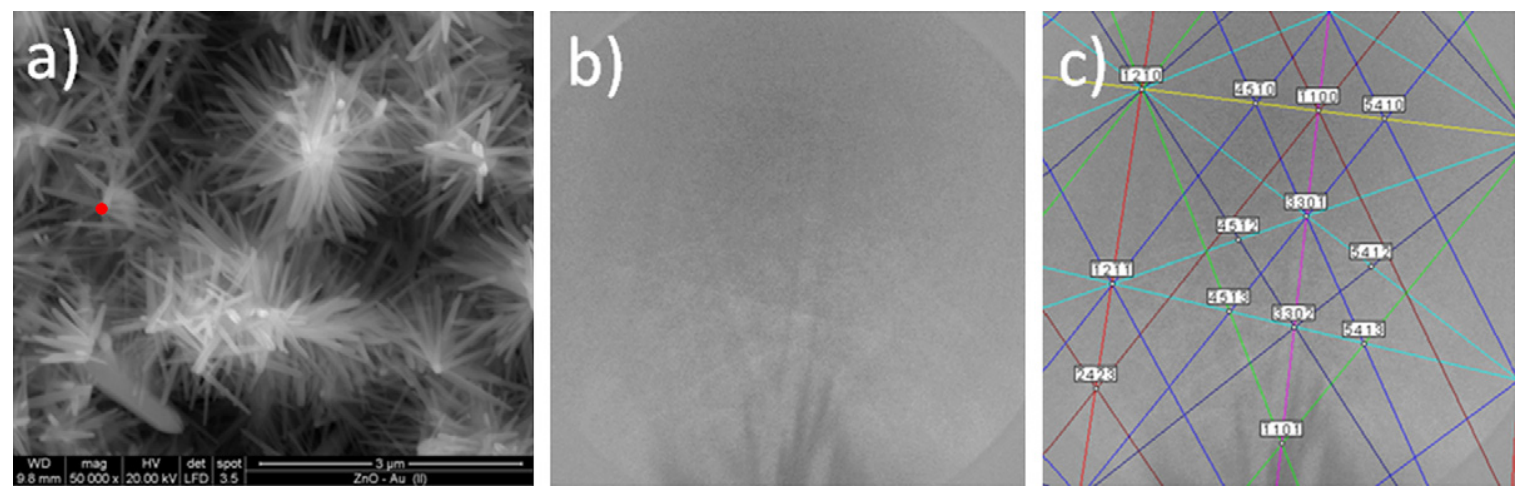

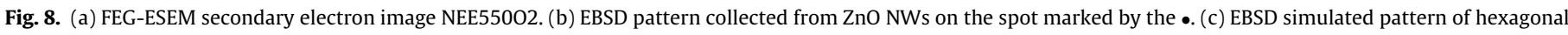
wurtzite structure overlaid on pattern of (b). 


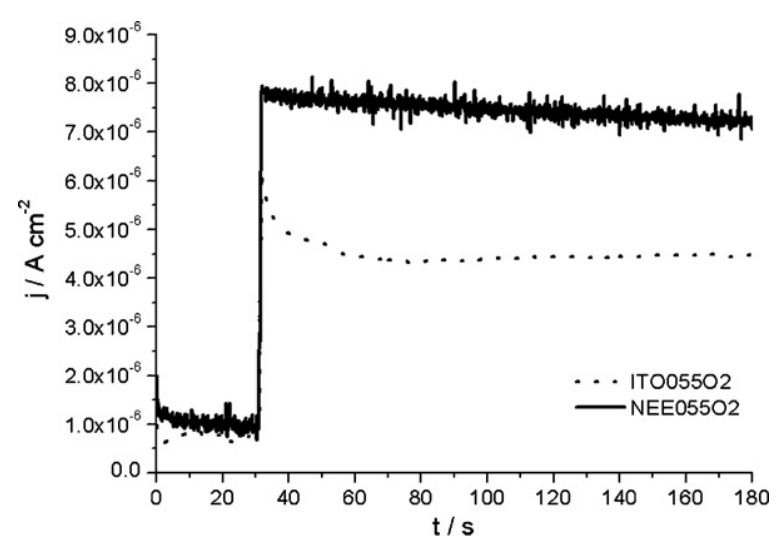

Fig. 9. Measured photocurrent density at $0.400 \mathrm{~V}$ vs. $\mathrm{Ag} / \mathrm{AgCl}$ sat. in $0.1 \mathrm{M}$ phosphate buffer solution pH 7. Dotted line ITO55002, straight line NEE550O2.

difference between the current measured in the dark and the plateau current recorded after $140 \mathrm{~s}$ of irradiation. Table 1 lists the photocurrent values (measured as described above) for all the prepared electrodes. As expected, ITO550N2 shows a really low photocurrent $\left(0.23 \mu \mathrm{A} \mathrm{cm}^{-2}\right)$, confirming that almost no $\mathrm{ZnO}$ has been deposited. ITO7000N2 and ITO550O2 achieved comparable photocurrents, namely $4.00 \mu \mathrm{Acm}^{-2}$ and $3.73 \mu \mathrm{Acm}^{-2}$, respectively; ITO70002 gives a photocurrent of $2.89 \mu \mathrm{A} \mathrm{cm}^{-2}$. This higher photocurrent at ITO700N2 and ITO550O2 can be related to their higher surface area, since they showed thinner and more dense ZnO NWs.

NEE550N2 and NEE55002 achieved comparable photocurrents which are double in comparison with the homologous electrodes deposited at $-0.700 \mathrm{~V}$, even if the total charge involved in the electrodeposition of the former is lower. This confirms that the morphology of the nanostructured materials is extremely important in defining its physico-chemical properties. On the other hand, it's important to note that the most efficiently branched electrode, namely NEE550O2, provides a photocurrent which is 50\% higher than the best ITO electrode (ITO55002). These results confirm that branched nanostructures improve significantly the photocatalytic performances, the overall effect being the result of a positive combination of improved electron transport efficiency and increased surface area.

The electrodes stability has been studied extending the photoelectrochemical experiments up to $120 \mathrm{~min}$. Only NEE055O2 and ITO055O2 electrodes were taken into account as representative for both structures, i.e. branched and linear nanofibres, respectively (Fig. 10).

ITO05502 shows a quite stable signal over time (after $2 \mathrm{~h}$ the photocurrent is $99 \%$ of the initial one), while, with NEE055O2, the photocurrent recorded after $2 \mathrm{~h}$ decreases of approximately 6-7\%. SEM observations performed after the prolonged photoelectrochemical tests did not show any detectable change in the structure of both electrodes (not shown). These evidences indicate that the slight decrease in photocurrent with time observed with the branched NEE is due more probably to the entrapment of small

Table 1

Photocurrent density of water oxidation for ZnO NWs grown on ITO and on 3D$\mathrm{NEE}$, reported in $\mu \mathrm{Acm}^{-2}$, at constant potential of $0.40 \mathrm{~V}$ in $0.1 \mathrm{M}$ phosphate buffer solution $\mathrm{pH} 7$.

\begin{tabular}{rlll}
\hline ITO550N2 & ITO55002 & ITO700N2 & ITO70002 \\
\hline 0.23 & 4.00 & 3.73 & 2.89 \\
NEE550N2 & NEE55002 & NEE700N2 & NEE70002 \\
\hline 5.58 & 6.30 & 1.98 & 2.72 \\
\hline
\end{tabular}

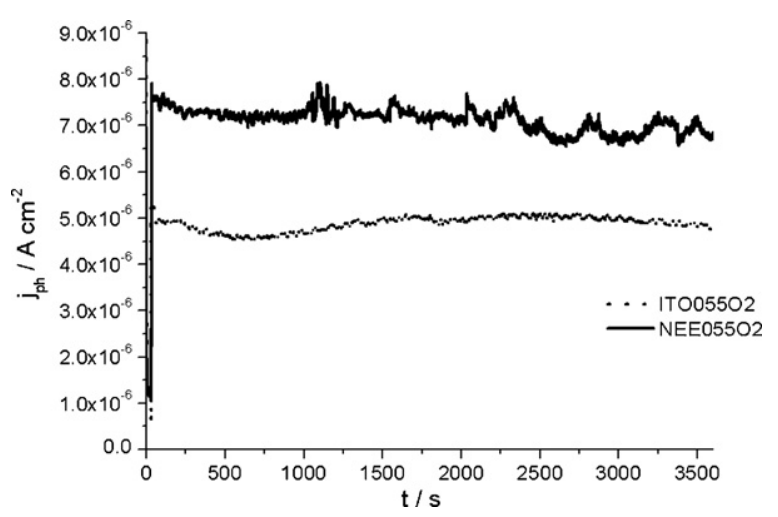

Fig. 10. Photocurrent stability measured at $0.400 \mathrm{~V}$ vs. $\mathrm{Ag} / \mathrm{AgCl}$ sat. in $0.1 \mathrm{M}$ phosphate buffer solution pH 7 over 2 h. Dotted line ITO550O2, straight line NEE550O2.

oxygen bubbles within the intricate structure of $\mathrm{ZnO}$ nanoneedles rather than to photochemical degradation of the $\mathrm{ZnO}$ nanofibers. Note that, in any case, the photocurrent density at the branched NEE is always significantly higher than that generated by the linear nanofibers on ITO. Further investigations on the role of gas evolution are presently in progress, looking on one side to the possibility to avoid or minimize the effect of gas entrapment and, on the other, by studying the use of the branched nanofibers for catalyzing photoelectrochemical reactions which do not generate gas.

\section{Conclusions}

This is the first time that $\mathrm{ZnO}$ branched nanostructures are obtained by a relatively simple combination of wet-chemistry methods (which include the chemical growth of substrate NWs, in this case $\mathrm{Au}$ in nanoporous templating membranes), combined with the electrochemical growth of $\mathrm{ZnO}$ sub-nanostructures. Notwithstanding its easiness, the method shows to be effective in improving the photoelectrochemical performances of the hierarchically branched nanoelectrodes, indicating interesting prospects for their photoelectrochemical application. Interestingly, 3D-NEE showed improved catalytic properties both on the electrochemical reactions involved in the deposition and for the controlled growth of the ZnO NWs substructures.

Finally, we demonstrated that EBSD constitutes a new efficient analytical tool for phase identification analysis of complex nanostructures such as the hierarchical $\mathrm{ZnO}$ nanostructures prepared here. The obtained results support the application of this technique as a valuable alternative to XRD analysis, with the great advantage of obtaining precise structural information on individual nanostructures.

\section{Acknowledgements}

This research was financially supported by MIUR(Rome), project PRIN 2008MWHCO2. We thank Prof. Michela Signoretto (University Ca' Foscari of Venice) for helpful discussion and suggestion and Dr. Alessandro Patelli (CIVEN, Porto Marghera) for assistance with the plasma etching experiments.

\section{References}

[1] M.H. Huang, S. Mao, H. Feick, H. Yan, Y. Wu, H. Kind, E. Weber, R. Russo, P. Yang, Science 292 (2001) 1897.

[2] Q. Zhang, C.S. Dandeneau, X. Zhou, G. Cao, Advanced Materials 21 (2009) 1

[3] L.E. Greene, M. Law, D.H. Tan, M. Montano, J. Goldberger, G. Somorjai, P. Yang, Nano Letters 5 (2005) 1231.

[4] C. Ma, D. Moore, Y. Ding, J. Li, Z.L. Wang, International Journal of Nanotechnology 1 (2004) 431

[5] P.X. Gao, Z.L. Wang, Small 1 (2005) 945. 
[6] Z. Peng, G. Dai, P. Chen, Q. Zhang, Q. Wan, B. Zou, Materials Letters 64 (2010) 898.

[7] G. Centi, S. Perathoner, European Journal of Inorganic Chemistry 26 (2009) 3851.

[8] S.P. Albu, H. Tsuchiya, S. Fujimoto, P. Schmuki, European Journal of Inorganic Chemistry 27 (2010) 4351.

[9] K. Shankar, J.I. Bashan, N.K. Allam, O.K. Varghese, G.K. Mor, X. Feng, M. Paulose, J.A. Seabold, K.-S. Choi, C.A. Grimes, Journal of Physical Chemistry C 113 (2005) 6327.

[10] G. Shen, Y. Bando, C.-J. Lee, Journal of Physical Chemistry B 109 (2005) 10779.

[11] J. Elias, R. Tena-Zaera, C. Lévy-Clément, Journal of Electroanalytical Chemistry 621 (2008) 171

[12] J. Cui, J. Gibson, Journal of Physical Chemistry B 109 (2005) 22074.

[13] F.J. Sheini, I.S. Mulla, D.S. Joag, M.A. More, Thin Solid Films 517 (2009) 6605.

[14] B. Canava, D. Lincot, Journal of Applied Electrochemistry 30 (2000) 711.

[15] A. Goux, T. Pauportè, J. Chivot, D. Lincot, Electrochimica Acta 50 (2005) 2239.

[16] F. Xu, Y. Lu, Y. Xie, Y. Liu, Journal of Solid State Electrochemistry 14 (2010) 63.

[17] L. Xu, Q. Chen, D. Xu, Journal of Physical Chemistry C 111 (2007) 11560.

[18] X.-J. Wu, F. Zhu, C. Mu, Y. Liang, L. Xu, Q. Chen, R. Chen, D. Xu, Coordination Chemistry Reviews 254 (2010) 1135.

[19] J. Elias, C. Lévy-Clément, M. Bechelany, J. Michler, G.-Y. Wang, Z. Wang, L. Philippe, Advanced Materials 22 (2010) 1607.

[20] V.P. Menon, C.R. Martin, Analytical Chemistry 67 (1995) 1920

[21] P. Ugo, L.M. Moretto, in: C. Zoski (Ed.), Handbook of Electrochemistry, Elsevier, Amsterdam, 2007 (Chapter 16)

[22] S. Yu, J. Wharton, N. Li, C.R. Martin, Nano Letters 3 (2003) 815.

[23] M. De Leo, A. Kuhn, P. Ugo, Electroanalysis 19 (2007) 227.

[24] K.Z. Baba-Kishi, Journal of Materials Science 37 (2002) 1715.
[25] A. Gambirasi, S. Cattarin, M. Musiani, L. Vàzquez-Gòmez, E. Velato, Electrochimica Acta 56 (2011) 8582.

[26] B. Brunetti, P. Ugo, L.M. Moretto, C.R. Martin, Journal of Electroanalytical Chemistry 491 (2000) 166.

[27] M. De Leo, F.C. Pereira, L.M. Moretto, P. Scopece, S. Polizzi, P. Ugo, Chemistry of Materials 19 (2007) 5955.

[28] P. Ugo, L.M. Moretto, F. Vezzà, ChemPhysChem 3 (2002) 917.

[29] P. Ugo, in: C.A. Grimes, E.C. Dickey, M.V. Pishko (Eds.), Encyclopedia of Sensors, American Scientific Publishers, Stevenson Ranch, 2005.

[30] M.R. Khajavi, D.J. Blackwood, G. Cabanero, R. Tena-Zaera, Electrochimica Acta 69 (2012) 181

[31] J. Elias, R. Tena-Zaera, C. Lévi-Clément, Thin Solid Films 515 (2007) 8553.

[32] D. Ito, M.L. Jespersen, J.E. Hutchinson, ACS Nano 2 (2008) 2001.

[33] J.B. Baxter, E.S. Aydil, Applied Physics Letters 86 (2005) 053114.

[34] A.R. Schwarzer, D.P. Field, B.L. Adams, M. Kumar, A.J. Schwartz, in: A.J. Schwartz, M. Kumar, B.L. Adams, D.P. Field (Eds.), Electron Backscatter Diffraction in Materials Science, Springer, 2009.

[35] J.A. Small, J.R. Michael, Journal of Microscopy 201 (2001) 59.

[36] J.A. Small, J.R. Michael, D.S. Bright, Journal of Microscopy - Oxford 206 (2002) 170.

[37] J.P. Long, B.S. Simpkins, D.J. Rowenhorst, P.E. Pehrsson, Nano Letters 7 (2007) 831.

[38] A. Gambirasi, S. Cattarin, M. Musiani, L. Vázquez-Gómez, E. Verlato, Electrochimica Acta 56 (2011) 8582.

[39] Y.N. Picard, L. Mazeina, S. Maximenko, J.A. Freitas, S.M. Prokes, M.E. Twigg Microscopy and Microanalysis 15 (Suppl. 2) (2009) 402. 